\title{
Flexibility of Short-Strand RNA in Aqueous Solution as Revealed by Molecular Dynamics Simulation: Are A-RNA and A'-RNA Distinct Conformational Structures?
}

\author{
Defang Ouyang ${ }^{1,2}$, Hong Zhang ${ }^{2}$, Dirk-Peter Herten ${ }^{3}$, Harendra S. Parekh ${ }^{1}$, Sean C. \\ $\operatorname{Smith}^{2 *}$ \\ ${ }^{1}$ School of Pharmacy,
}

The University of Queensland, Brisbane, QLD 4072, Australia.

${ }^{2}$ Centre for Computational Molecular Science,

Australian Institute of Bioengineering and Nanotechnology,

The University of Queensland, Brisbane, QLD 4072, Australia.

${ }^{3}$ BIOQUANT, Universität Heidelberg, Im Neuenheimer Feld 267, 69120 Heidelberg, Germany.

*Corresponding author. Tel: +61-7-3346 3949; Fax: +61-7-3346-3992; Email:

s.smith@uq.edu.au

Submitted to <Aust. J. Chem> 


\section{Abstract}

We use molecular dynamics simulations to compare the conformational structure and dynamics of a 21-base pair RNA sequence initially constructed according to the canonical A-RNA and A'-RNA forms in the presence of counterions and explicit water., Our study aims to add a dynamical perspective to the solid-state structural information that has been derived from X-ray data for these two characteristic forms of RNA. Analysis of the three main structural descriptors commonly used to differentiate between the two forms of RNA - namely major groove width, inclination and the number of base pairs in a helical twist - over a 30ns simulation period reveals a flexible structure in aqueous solution with fluctuations in the values of these structural parameters encompassing the range between the two crystal forms and more. This provides evidence to suggest that the identification of distinct A-RNA and A'-RNA structures, while relevant in the crystalline form, may not be generally relevant in the context of RNA in the aqueous phase. The apparent structural flexibility observed in our simulations is likely to bear ramifications for the interactions of RNA with biological molecules (e.g. proteins) and non-biological molecules (e.g. non-viral gene delivery vectors). 


\section{Introduction}

RNA molecules are critical to many biological processes in life science, the most reported of which include the transmission of genetic information, the catalysis of biochemical reactions, RNA inference in eukaryotes and a structural role in cellular organelles ${ }^{1-5}$. The 2'-hydroxyl (2'-OH) group is the main difference between RNA and DNA and plays a fundamental role in both RNA structure and function. In general, unlike the regular conformation of DNA, such as A-, B- or Z-DNA, RNA structures are strikingly diverse, from linear duplex RNA, hairpin molecules to loop structures ${ }^{6-10}$. Among RNA double-helices, two major right-handed conformations: the 11-fold helix of A-RNA and 12-fold helix of A'-RNA have been identified experimentally in crystal structures ${ }^{11-16}$. The major A-RNA conformation is apparent in natural RNA polynucleotides, or when crystallized from solutions of low ionic strength, while A'-RNA is formed from higher ionic strength solutions. ${ }^{17}$ The two forms of RNA retain the same overall helical features, however A'-RNA possesses a wider major groove than that of A-RNA. The details of their structural characteristics are discussed in several texts about nucleic acid structures. ${ }^{11-13}$ For example, from Protein Database Bank (PDB) and Nucleic Acid Database (NDB) survey data, the following three RNA molecules have been found to adopt the structural features of A'-RNA: the tridecamer r(UGAGCUUCGGCUC), the RNA dodecamer duplex (r-GGACUUCGGUCC) $)_{2}$ and helix IV of 5S rRNA. ${ }^{16,18,19}$ For later reference the helical parameters, groove widths and torsion angles of these structures are provided in Table 1, computed utilizing two common structural analysis programs 3DNA ${ }^{20}$ and CURVES. ${ }^{21,22}$ 
Understanding both the structure and dynamics of RNA molecules on the atomistic level is critical to gaining a clearer picture of their role and function as therapeutic agents in gene silencing. This challenge can be addressed by a combination of experimental approaches and molecular dynamics simulations. In recent years, a significant number of molecular dynamics studies ${ }^{23-30}$ on DNA and RNA systems have appeared. Cheatham \& Kollman reviewed molecular dynamics simulations of nucleic acids from 1995 to 2000, discussing force fields for nucleic acids and simulation protocols using explicit solvent and counterions. ${ }^{30,31}$ Computational studies of DNA and RNA have also been elaborated in the recent book by Sponer and Lankas. ${ }^{32}$ In relation to molecular dynamics simulations of RNA specifically, progress made in the last twenty years in this area has been summarized by McDowell et al. ${ }^{33}$ and by Hashem et al., ${ }^{34}$ providing summaries of simulations, force field approximations, RNA interactions with solvents and ions, catalytic RNAs and RNA-protein (or small molecule) complexes. tRNA was the first RNA molecule with an X-ray structure to be studied by MD simulations. ${ }^{35,36}$ Although the first simulation of tRNA was quite simple without explicit treatment of solvent, counterions and hydrogen atoms of the backbone, it was an important step towards the study of RNA molecules using the MD simulations. More recent studies have covered longer timescales with stable simulations of solvated and neutralized systems. ${ }^{37-43}$ Comparative studies of duplex RNA and DNA have revealed a striking contrast in structural flexibility. ${ }^{44,45}$ Attention has been focused both through experiment and simulation ${ }^{46}$ on the phenomenon of base pair opening and closing, which has been found to occur much more readily in RNA than in DNA. In addition, the opening mechanism of wobble pairs $d(G \cdot T)$ and $r(G \cdot U)$ in DNA and RNA duplexes was investigated with a 
combination of imino proton exchange and molecular dynamics simulation. ${ }^{47}$ The results indicated that the opening of wobble pairs is the rotation of bases toward the major groove. Further recent progress has been discussed by Hall. ${ }^{48}$

While as summarized above there have been a number of MD simulations for RNA, studies of the dynamical properties of A'-RNA are apparently still rare. By virtue of its characteristic 12-fold helix, the wider major groove of A'-RNA could be considered to bear some ramifications with respect to the propensity for complexing with other proteins, vectors or drugs. It is however worthy of note that the structures of A-RNA and A'-RNA have only been distinguished as independently stable conformations in the context of crystal structures, although the NUCGEN module of AMBER facilitates the construction of both forms with any given RNA sequence. In this paper, we use molecular dynamics simulations to compare the conformational structure and dynamics of a 21-base pair RNA sequence initially constructed according to the canonical A-RNA and A'-RNA forms in the presence of counterions and explicit water., in order to add a dynamical perspective relevant for the aqueous phase beyond the static perspective that has been derived from X-ray data. The original motivation was to explore whether a priori one might expect an A'-RNA conformation to display contrasting complexation propensities with other biomolecules in comparision with A-RNA due to its apparently wider major groove. The first step in examining this question was to analyse the fluctuations in major groove width for the two forms of RNA in aqueous solution. As will be seen below, the marked structural flexibility of RNA reveals itself yet again in our simulations - to the extent that we cannot find any evidence on the basis of our MD simulations that A-RNA and 
A'-RNA are indeed distinguishable stable conformations in solution - at least for the specific RNA strand studied herein. We have additionally examined the phenomenon of base pair opening, revealing base-pair opening and closing events for both r(A-U) and $\mathrm{r}(\mathrm{G}-\mathrm{C})$ base pairs on timescales consistent with that found in the earlier work of MacKerrel and $\operatorname{Pan}^{46}$ using the CHARMM forcefield (in contrast to the present AMBER ff99 force field) and again with no marked contrast between simulations that started with the A-RNA and the A'-RNA forms respectively.

\section{Simulation details}

The sequence of the 21 base pair siRNA is taken from the earlier study by Putral et al ${ }^{49}$ and is as follows:

Sense 5'- GCAACAGUUACUGCGACGUUU-3'

Antisense 3'- UUCGUUGUCAAUGACGCUGCA -5'

The present MD simulations used the AMBER9 software package with the all-atom AMBER99 force field (ff99) for RNA. Both 3'-terminal UU of the RNA duplex were cut to form a canonical RNA duplex and then A-RNA and A'-RNA was generated in the NUCGEN module of AMBER. The electrostatic interactions were calculated with the particle mesh Ewald method and a cutoff of $10 \AA$ was employed. Using the LEAP module in AMBER, $\mathrm{Na}^{+}$counterions were added to the RNA structure to neutralize the negative charge and the whole system was then immersed in a truncated octahedral water box with a solvation shell of $8 \AA$ thickness using TIP3P model for water. This procedure resulted in solvated structures containing approximately 30000 atoms which include the 1215 RNA atoms, 36 counterions $\left(\mathrm{Na}^{+}\right)$, and the remainder were water molecules in a single simulation box $18.774 \AA$ An length. 
The minimization procedure for solvation of RNA consisted of two steps. In the first step, the RNA part was fixed while minimizing the positions of the water and ions. The solvated structures were then subjected to 500 steps of steepest descent minimization followed by 500 steps of conjugate gradient minimization. During this minimization the RNA molecule was held fixed in its starting conformations using harmonic constraints with a force constant of $500 \mathrm{kcal} / \mathrm{mol} / \AA^{2}$. In the second stage, the entire system was minimized by 2000 steps of steepest descent minimization followed by 8000 steps of conjugate gradient minimization without the restraints.

The optimized structure was then subjected to 20 ps of MD, using a 2 fs time step for integration, during which the system was gradually heated from 0 to $300 \mathrm{~K}$ using 10 $\mathrm{kcal} / \mathrm{mol} / \AA^{2}$ weak positional restraints on the RNA. The SHAKE algorithm was used in which all bonds involving hydrogen are constrained. After the system was heated at constant volume with weak restraints on RNA, the main MD simulation was performed for $30 \mathrm{~ns}$ with a time step of 2 fs under constant pressure (average pressure 1atm) and constant temperature $(T=300 \mathrm{~K})$ conditions (i.e., an NPT ensemble) without positional restraints. The random number seed was changed at each restart ${ }^{50,51}$ and periodic boundary conditions were employed in three dimensions. Isotropic position scaling was used to maintain the pressure and a relaxation time of 2 ps was used. SHAKE was used to constrain bonds involving hydrogen and the temperature was kept at $300 \mathrm{~K}$ with the Langevin dynamics using a collision frequency of $1.0 \mathrm{ps}^{-1}$. 


\section{Results and discussion}

We begin with a consideration of two different common approaches to characterizing the structural parameters of our RNA. As noted in the introduction above, groove widths, helical parameters and torsional angles were calculated for the three A'-RNA structures obtained from PDB and NDB databases with two commonly used programs, namely CURVES5.1 ${ }^{21,22}$ and 3DNA (Table 1). ${ }^{20}$ The major groove width from 3DNA is calculated in terms of refined phosphate-phosphate distances, which take into account the directions of sugar-phosphate backbones. Given the implicit definitions in the two programs, for comparison purposes the final width from 3DNA is shifted by a subtraction of $5.8 \AA$ from the original values. ${ }^{52}$ It can be seen from the comparison of the structural parameters calculated by the two programs in Table 1 that most parameters are similar. However, the values of the inclination angles and the major or minor groove widths are quite different. For example, the inclination angle of the 413D RNA sequence changes from $-5^{\circ}$ computed via CURVES to $12.8^{\circ}$ computed via 3DNA, while its average major groove width (i.e., averaged along the strand) is about 8 and $11 \AA$, respectively. The other two sequences in Table 1 show similar consistencies and contrasts. As has also been pointed out previously, ${ }^{53-55}$ the two programs produce different values of helical and base-pair parameters, especially on distorted and irregular RNA molecules. Lu and Olson have demonstrated that the main cause of conflicting structural interpretations from the different programs relates to the reference frames used by each program. ${ }^{54}$ To resolve the numerical discrepancies, in 1999, the Tsukuba Workshop on nucleic acid structure and interactions reached an agreement with "a standard reference frame for the description of nucleic acid base-pair geometry". ${ }^{56}$ In accord with this, the 3DNA program with this 
standard reference frame is utilized for the data analysis below.

To explore the conformational dynamics, two separate MD simulations were seeded with canonical A-RNA and A'-RNA structures respectively and run over a period of 30ns. Plots shown in Fig.1 of the RMSD for both forms behave similarly over the 30 ns simulation and the oscillations about a stable mean from $c a$. 3ns onwards indicate that the simulations are stable over this period. Figs. $2 \mathrm{a}$ and $2 \mathrm{~b}$ provide stereo views of both the canonical structures and the time-averaged MD structures of the A-RNA and the A'-RNA respectively. Corresponding structural data associated with these figures is provided in Table 2. Comparison of two average structures indicates that they are broadly similar to each other. It is relevant to point out that a number of the structural parameters in Table 2 display quite large standard deviations (SDs) - a measure of the magnitude of fluctuations over the time of the simulation. We should stress here that these large SDs do not imply that the simulation is not equilibrated, since they do not decrease over time. Rather they are indicators of intrinsic structural flexibility. This leads one to examine the basis for distinguishing between the A- and A'-RNA structures in more detail.

Our principal strategy in analyzing the dynamics is to examine the three main structural parameters that are commonly used to distinguish A-RNA from A'-RNA in crystal data, namely major groove width, inclination and the number of base pairs per helical twist. We compute these paramaters for snapshots of the RNA system at 1ns intervals along each trajectory, the results of which are presented in Fig. 3. In Fig. 3a the major groove width is plotted as a function of time for both simulations. Also indicated by arrows 
against the vertical axis are the "canonical" values generated by the NUCGEN module of AMBER9 (i.e., prior to structure optimization and thermalisation), which are based on collective consideration of various crystal structures. A comparison of the $t=0$ starting values for the MD with the canonical values generated by NUCGEN reveals that even during the relaxation stages both RNA strands attain significantly larger groove widths than the typical crystal-packing values as represented by the arrows - presumably relating to the intrinsic differences between the crystal and the aqueous phases (i.e., different amounts of water, the lack of there-dimensional periodic packing forces in the aqueous phase as compared with the crystal, etc.). In Fig. $3 b$ we present the values for the inclination parameter, computed similarly at ns intervals along each trajectory. Finally in Fig. $3 \mathrm{c}$ is shown the number of base pairs per helical twist, evaluated by manual inspection of the structures at ns intervals. From inspection of Figs 3a-c it becomes immediately apparent that the conformational fluctuations of both the "A-RNA" and the "A'-RNA" simulations are significant, encompassing in each case ranges wider than the initial difference between the two. Also indicated in Figs. $3 \mathrm{a}$ and $3 \mathrm{~b}$ are the standard deviation (SD) estimates for the major groove width and inclination parameters (vertical bars on each point). These SDs, which are also quite significant in magnitude, relate to the fact that the groove width and inclination are evaluated with respect to individual base pairs and then averaged along the strand. Hence, the SDs are a reflection of the structural variability along the strands at any given time. Thus, both from the point of view of intra-strand structural heterogeneity and time-dependent behaviour, collective consideration of the results in Figs3a-c leads to the suggestion that the MD simulations in aqueous solution for the A-RNA and A'-RNA structures show no evidence that these can 
be regarded as distinct conformational structures.

One could potentially question whether there is some aspect lacking in the forcefield that we have utilized here (AMBER ff99) that prevents it from reliably simulating stable A-RNA and A'-RNA conformers. However, there are at least two factors lending confidence that the observations derived from this work are plausible. Firstly we note that our finding is not inconsistent with the fact that, to the best of our knowledge, the evidence for these two distinct conformations derives to date only from X-ray studies of the crystalline state. Secondly, our results appear to be broadly consistent with features seen previously in the simulations of Pan and MacKerell, ${ }^{46}$ in their comparative study of RNA and DNA dynamics using the CHARMM27 forcefield. Their 5-7ns simulations were carried for a series of shorter RNA strands (8-12 base pairs) and hence do not admit comparison of helical structural parameters such as those characterized here in Fig.3. Rather, they focused on the phenomenon of base pair opening, i.e., localized cleavage of the Watson-Crick hydrogen bonded base pairing. Their simulations revealed base pair opening events lasting for periods from a few ps to a few ns before the hydrogen bond was re-established. This phenomenon, which occurred more rarely and with much shorter open state lifetimes in their DNA simulations, was found to occur for both $r(U \cdot A)$ and r(G.C) pairs. We observe the same phenomenon in our simulations as exemplified in Fig. 4, which plots the N1-N3 hydrogen bond distance as a function of simulation time for $\mathrm{r}(\mathrm{U} \cdot \mathrm{A})$ and $\mathrm{r}(\mathrm{G} \cdot \mathrm{C})$ pairs selected close to the end of the strand and also in the middle. The spikes in the main plot (some representative examples are shown in higher resolution in the insets) correspond to opening events in which the hydrogen bonds were stretched 
beyond $4.5 \AA$ for longer than 10 ps.

The conformational flexibility of RNA revealed in this work (and foreshadowed in part by the earlier simulations of Pan and MacKerell ${ }^{46}$ ) may be a significant factor in relation to its structural diversity and biological function. Generally speaking, a wide major groove should enable it to easily interact with proteins, while a narrow one would tend to obstruct such interactions. For instance, helix IV of 5S rRNA has a wide major groove due to the combination of cross-strand purine stacks, in which the major groove width is $11 \AA .{ }^{19}$ Furthermore, the major groove of helix IV is wide enough to accommodate an interacting protein, such as the ribosomal protein $\mathrm{L} 25$, which can be used for RNA recognition. Another interesting example is base-pair dynamics in sarcin-ricin domain RNA ${ }^{61}$. The domain is a universal element of the large subunit ribosomal RNA. The sarcin-ricin domain is necessary for binding elongation factors and can be specifically cleaved by two ribotoxins $\alpha$-sarcin and ricin. The imino proton exchange experiments indicated the presence of opening and closing dynamics for a selected base pair of the active area, which suggested that these opening conformational changes might be significant for the recognition of RNA by the two toxins. The present simulations add a dynamical context to this general understanding - highlighting that structural parameters such as major groove width are remarkably flexible in short RNA strands - potentially allowing them to adapt on the ps to ns timescale during encounter and interaction with complexing partners. 


\section{Conclusions}

Among RNA double-helices, two major right-handed conformations: the 11-fold helix of A-RNA and 12-fold helix of A'-RNA have been identified experimentally in crystal structures ${ }^{11-16}$. The major A-RNA conformation is apparent in natural RNA polynucleotides, or when crystallized from solutions of low ionic strength, while A'-RNA is formed from higher ionic strength solutions. ${ }^{17}$ These two forms of RNA retain the same overall helical features, however A'-RNA holds wider major groove than that of A-RNA. In this work we have carried out 30ns MD simulations using the AMBER ff99 forcefield to explore the structural and dynamical features of A-RNA and A'-RNA forms of the same short strand sequence in an aqueous environment. Monitoring the three major structural parameters commonly used to distinguish the A- and A'- RNA conformers as a function of time, namely the major groove width, the inclination and the number of base pairs per helical twist, we find that these descriptors fluctuate significantly for both simulations, covering in each case a range at least as great or larger than the initial difference between the canonical A-RNA and A'-RNA forms. This indicates substantial flexibility in the RNA structure in the aqueous environment, to the extent that - at least for the specific strand studied here - we can find no evidence that the A-RNA and A'-RNA can be regarded as independently stable and distinct conformers. The implication of this is that the two forms may in fact be stable only in the presence of the appropriate crystal packing forces - an observation not inconsistent with the fact that the extant data characterizing the two forms is derived only from the crystalline states of various RNA strands. We find similar base-pair opening dynamics on comparable timescales to the earlier study of Pan and MacKerell based on the CHARMM forcefield ${ }^{46}$ 
- again emphasizing the flexibility of short RNA strands in water and lending some confidence that our simulations do not suffer from artifacts related to one specific force field. The observed fluctuation of the major groove width may have significance for the interactions of RNA with proteins and organic compounds. The interaction of various dendritic polycations utilized in gene delivery with RNA is currently being explored with a view to better understanding their interactions with RNA at the atomistic level.

\section{Acknowledgements}

We acknowledge generous allocations of CPU time from the Australian National Computing Infrastructure (NCI) facility as well as from the computational molecular science cluster computing facility at The University of Queensland, funded in part by the Australian Research Council (ARC) Linkage, Infrastructure, Equipment and Facilities (LIEF) scheme (grant number LE0882357). 


\section{References}

(1) Guerriertakada, C.; Gardiner, K.; Marsh, T.; Pace, N.; Altman, S. Cell 1983, 35, 849-857.

(2) Fire, A.; Xu, S.; Montgomery, M. K.; Kostas, S. A.; Driver, S. E.; Mello, C. C. Nature 1998, 391, 806-11.

(3) Flores, R.; Delgado, S.; Gas, M. E.; Carbonell, A.; Molina, D.; Gago, S.; De la Pena, M. In 29th Congress of the Federation-of-European-Biochemical-Societies; Elsevier Science Bv: Warsaw, POLAND, 2004, p 42-48.

(4) Frank, J.; Spahn, C. M. T. Reports on Progress in Physics 2006, 69, 1383-1417.

(5) He, L.; Hannon, G. J. Nature Reviews Genetics 2004, 5, 522-531.

(6) Voet, D. V., J.G. Pratt, C.W. Foundamentals of biochemistry: life at the molecular level; 3rd ed.; Wiley:

Hoboken, NJ, 2008.

(7) Theimer, C. A.; Finger, L. D.; Feigon, J. Rna-a Publication of the Rna Society 2003, 9, 1446-1455.

(8) Popenda, L.; Adamiak, R. W.; Gdaniec, Z. Biochemistry 2008, 47, 5059-5067.

(9) Schmitz, M.; Tinoco, I. Rna-a Publication of the Rna Society 2000, 6, 1212-1225.

(10) Nomura, Y.; Kajikawa, M.; Baba, S.; Nakazato, S.; Imai, T.; Sakamoto, T.; Okada, N.; Kawai, G. Nucleic Acids Res 2006, 34, 5184-5193.

(11) Neidle, S. Oxford Handbook of Nucleic Acid Structure Oxford University Press, 1999.

(12) Neidle, S. Principles of nucleic acid structure; Elsevier, 2008.

(13) Blackburn, G. M. E. Nucleic acids in chemistry and biology 3rd ed.; RSC Pub: Cambridge, UK 2006.

(14) Arnott, S.; Fuller, W.; Hodgson, A.; Prutton, I. Nature 1968, 220, 561-\&.

(15) Arnott, S.; Hukins, D. W. L.; Dover, S. D. Biochemical and Biophysical Research Communications 1972, 48, 1392-\&.

(16) Tanaka, Y.; Fujii, S.; Hiroaki, H.; Sakata, T.; Tanaka, T.; Uesugi, S.; Tomita, K.; Kyogoku, Y. Nucleic Acids Res 1999, 27, 949-955.

(17) Arnott S, H. D., Dover SD, Fuller W, Hodgson AR. J Mol Biol. 1973, 81, 107-122.

(18) Holbrook, S. R.; Cheong, C. J.; Tinoco, I.; Kim, S. H. Nature 1991, 353, 579-581.

(19) Correll, C. C.; Freeborn, B.; Moore, P. B.; Steitz, T. A. Cell 1997, 91, 705-712.

(20) Lu, X. J.; Olson, W. K. Nucleic Acids Res 2003, 31, 5108-5121.

(21) Lavery, R.; Sklenar, H. Journal of Biomolecular Structure \& Dynamics 1988, 6, 63-91.

(22) Lavery, R.; Sklenar, H. Journal of Biomolecular Structure \& Dynamics 1989, 6, 655-667.

(23) Kawakami, Y.; Hopfinger, A. J. Anticancer Drug Des 1992, 7, 181-201.

(24) Schmitz, U.; Ulyanov, N. B.; Kumar, A.; James, T. L. J Mol Biol 1993, 234, 373-89.

(25) Langley, D. R. J Biomol Struct Dyn 1998, 16, 487-509.

(26) Roll, C.; Ketterle, C.; Faibis, V.; Fazakerley, G. V.; Boulard, Y. Biochemistry 1998, 37, 4059-70.

(27) Eriksson, M. A.; Nilsson, L. Eur Biophys J 1999, 28, 102-11.

(28) Varnai, P.; Lavery, R. J Am Chem Soc 2002, 124, 7272-3.

(29) Huang, W. H.; Zinchenko, A. A.; Pawlak, C.; Chen, Y.; Baigl, D. Chembiochem 2007, 8, 1771-4.

(30) Cheatham, T. E., 3rd; Kollman, P. A. Annu Rev Phys Chem 2000, 51, 435-71.

(31) Cheatham, T. E., 3rd; Young, M. A. Biopolymers 2000, 56, 232-56.

(32) Sponer, J.; Lankas, F. Computational studies of RNA and DNA; Springer: The Netherlands, 2006.

(33) McDowell, S. E.; Spackova, N.; Sponer, J.; Walter, N. G. Biopolymers 2007, 85, 169-184.

(34) Hashem, Y.; Auffinger, P. Methods 2009, 47, 187-197.

(35) Prabhakaran, M.; Harvey, S. C.; Mao, B.; McCammon, J. A. Journal of Biomolecular Structure \& Dynamics 1983, 1, 357-369.

(36) Harvey, S. C.; Prabhakaran, M.; Mao, B.; McCammon, J. A. Science 1984, 223, 1189-1191.

(37) Yu, H.; Mu, Y. G.; Nordenskiold, L.; Stock, G. Journal of Chemical Theory and Computation 2008, 4, 1781-1787.

(38) Mazier, S.; Genest, D. Biopolymers 2008, 89, 187-196.

(39) Deng, N. J.; Cieplak, P. Journal of Chemical Theory and Computation 2007, 3, 1435-1450.

(40) Nystrom, B.; Nilsson, L. J Biomol Struct Dyn 2007, 24, 525-36.

(41) Ding, F.; Sharma, S.; Chalasani, P.; Demidov, V. V.; Broude, N. E.; Dokholyan, N. V. Rna 2008, 14, 1164-73.

(42) Anderson, P. C.; Mecozzi, S. J Comput Chem 2006, 27, 1631-40.

(43) Mu, Y.; Stock, G. Biophys J 2006, 90, 391-9. 
(44) Snoussi, K.; Leroy, J. L. Biochemistry 2001, 40, 8898-8904.

(45) Lee, J. H.; Pardi, A. Nucleic Acids Research 2007, 35, 2965-2974.

(46) Pan, Y. P.; MacKerell, A. D. Nucleic Acids Research 2003, 31, 7131-7140.

(47) Varnai, P.; Canalia, M.; Leroy, J. L. Journal of the American Chemical Society 2004, 126, 14659-14667.

(48) Hall, K. B. Current Opinion in Chemical Biology 2008, 12, 612-618.

(49) Putral, L. N.; Bywater, M. J.; Gu, W.; Saunders, N. A.; Gabrielli, B. G.; Leggatt, G. R.; McMillan, N. A. Mol Pharmacol 2005, 68, 1311-9.

(50) Cerutti, D. S.; Duke, R.; Freddolino, P. L.; Fan, H.; Lybrand, T. P. Journal of Chemical Theory and Computation 2008, 4, 1669-1680.

(51) Wu, E. L.; Han, K. L.; Zhang, J. Z. H. Chemistry-a European Journal 2008, 14, 8704-8714.

(52) El Hassan, M. A.; Calladine, C. R. Journal of Molecular Biology 1998, 282, 331-343.

(53) Lu, X. J.; Babcock, M. S.; Olson, W. K. Journal of Biomolecular Structure \& Dynamics 1999, 16, 833-843.

(54) Lu, X. J.; Olson, W. K. Journal of Molecular Biology 1999, 285, 1563-1575.

(55) Barbic, A.; Crothers, D. M. Journal of Biomolecular Structure \& Dynamics 2003, 21, 89-97.

(56) Olson, W. K.; Bansal, M.; Burley, S. K.; Dickerson, R. E.; Gerstein, M.; Harvey, S. C.; Heinemann, U.; Lu, X. J.; Neidle, S.; Shakked, Z.; Sklenar, H.; Suzuki, M.; Tung, C. S.; Westhof, E.; Wolberger, C.; Berman, H. M. Journal of Molecular Biology 2001, 313, 229-237.

(57) Pan, Y. P.; MacKerell, A. Biophysical Journal 2004, 86, 145A-145A.

(58) Liu, L.; Botos, I.; Wang, Y.; Leonard, J. N.; Shiloach, J.; Segal, D. M.; Davies, D. R. Science 2008, 320, 379-381.

(59) Gan, J., Shaw, G., Tropea, J.E., Waugh, D.S., Court, D.L., Ji, X. Mol.Microbiol 2007, 67, 143-154

(60) Gan, J. H.; Tropea, J. E.; Austin, B. P.; Court, D. L.; Waugh, D. S.; Ji, X. H. Cell 2006, 124, 355-366.

(61) Chen, C.; Jiang, L.; Michalczyk, R.; Russu, I. M. Biochemistry 2006, 45, 13606-13613. 


\section{Figure Captions}

Figure 1. Root mean square deviation (RMSD) of A-RNA (red line) and A'-RNA (black line).

Figure 2. Stereo views of the starting and averaged structures of (a) A-RNA and (b) A'-RNA.

Figure 3. (a) Plot of major groove width (averaged over strand length, standard deviation indicated by vertical bar) as a function of time for A-RNA (red line) and A'-RNA (black line); (b) Plot of inclination angle (averaged over strand length, standard deviation indicated by vertical bar) as a function of time for A-RNA (red) and A'-RNA (black); c) Number of base pairs per helical twist versus time for A-RNA (red) and A'-RNA (black).

Figure 4. Plot of N1-N3 distance of base pair versus time for A-RNA (red) and A'-RNA (black): a) r(U16.A23) base pair; b) r(U10·A29) base pair; c) r(G15.C24) base pair; d) r(G9.C30) base pair. (only the simulations with base opening events of longer than 10 ps and higher than $4.5 \AA$ are included.)

Note: The numbering scheme for the bases is as follows:

5'- G $_{20} \mathrm{CAACAGUUA}_{29} \mathrm{C}_{30} \mathrm{UGCGACGU}_{38}-3^{\prime}$

3'- $\mathrm{C}_{19} \mathrm{GUUGUCAAU}_{10} \mathrm{G}_{09}$ ACGCUGCA $_{01}-5^{\prime}$ 


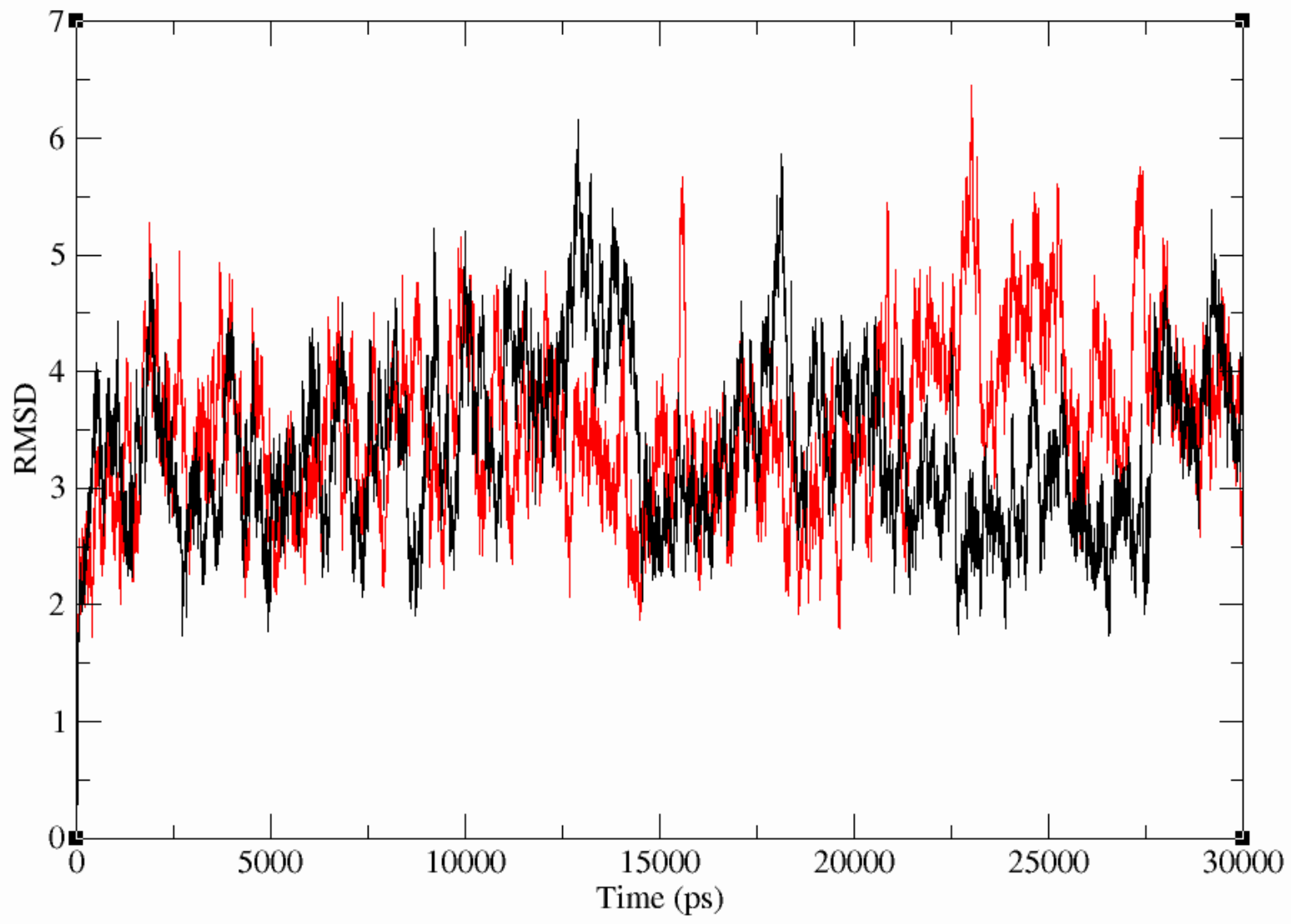

Figure 1 
(a)
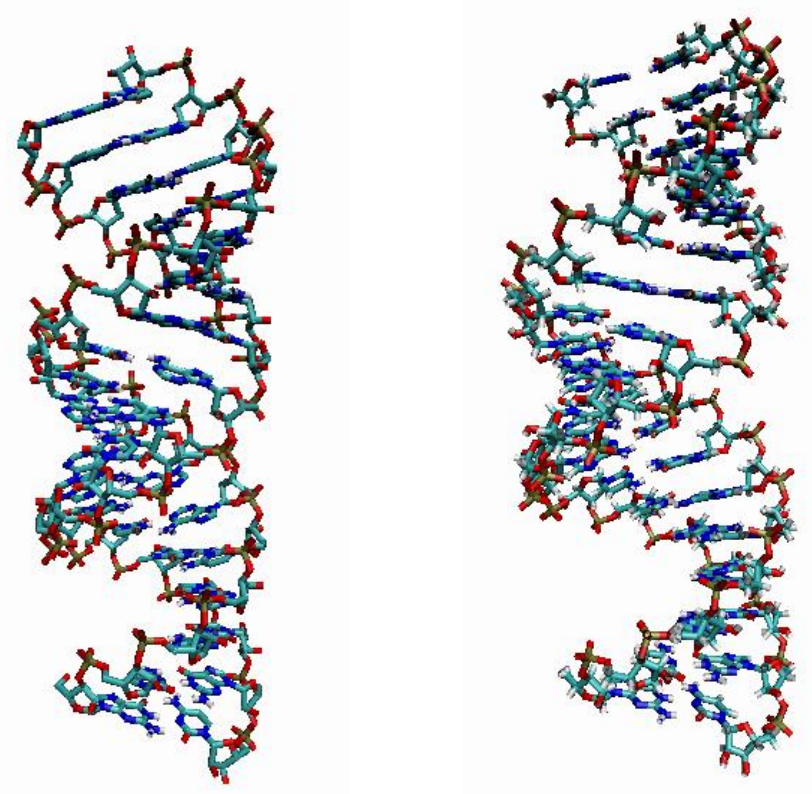

(b)
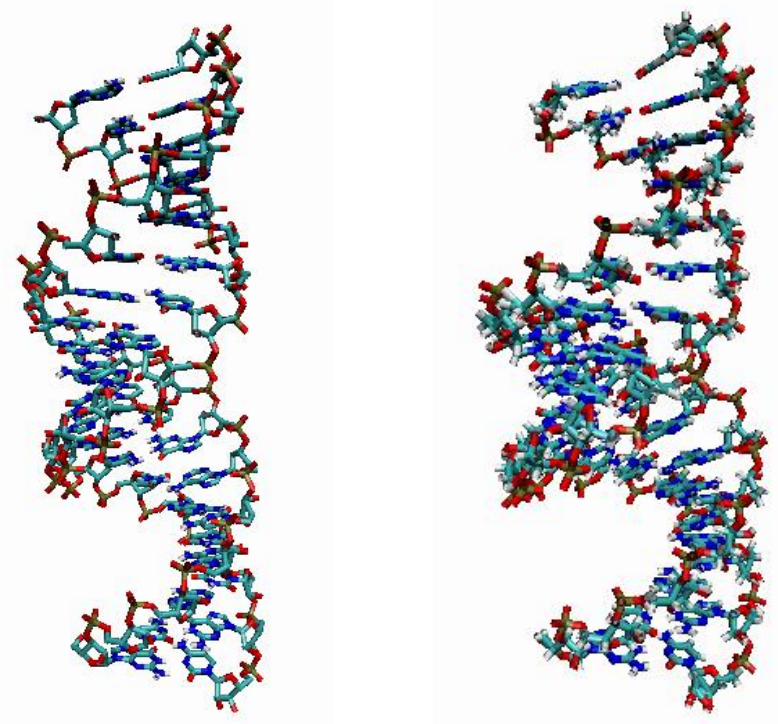

Figure 2 


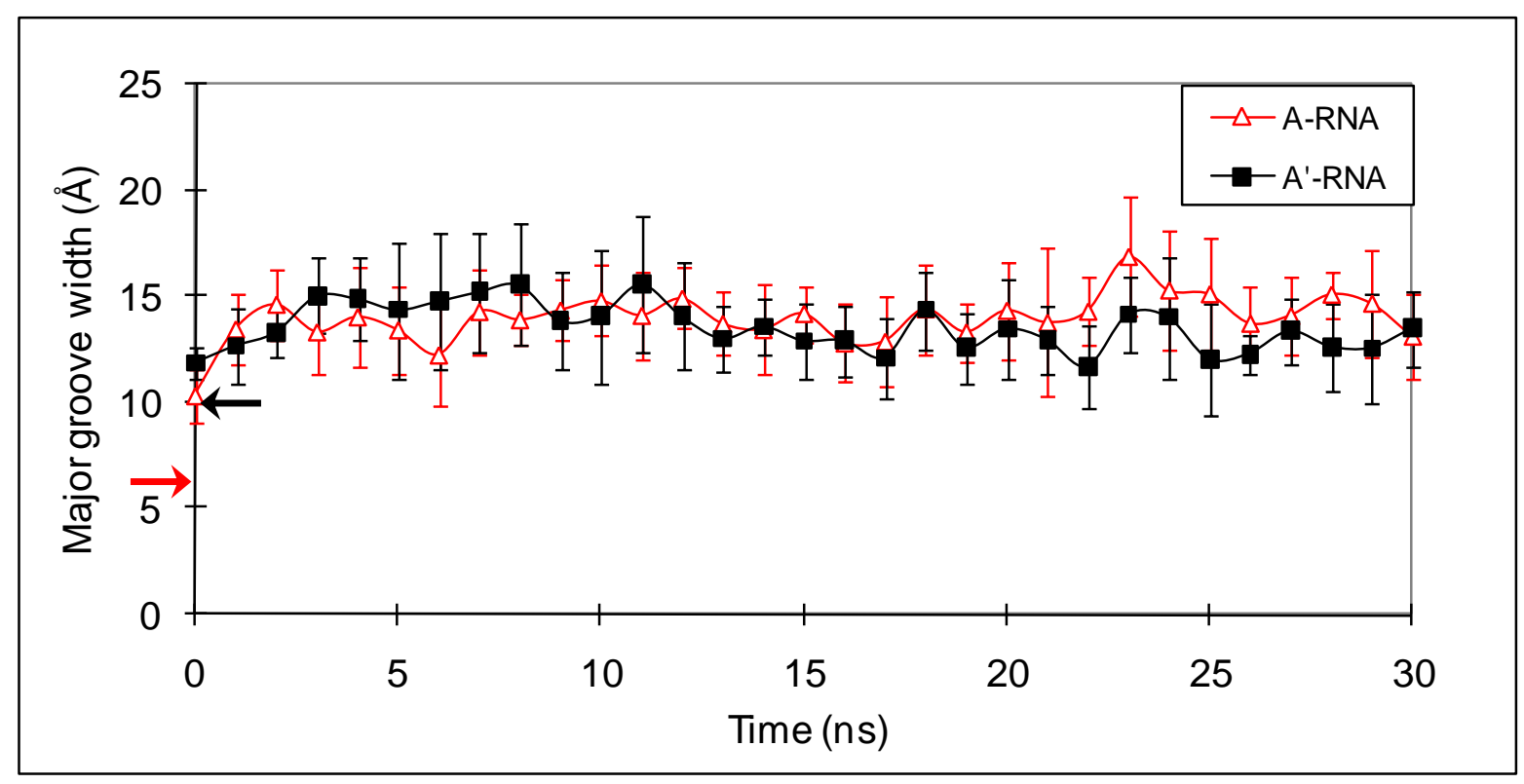

Figure 3a

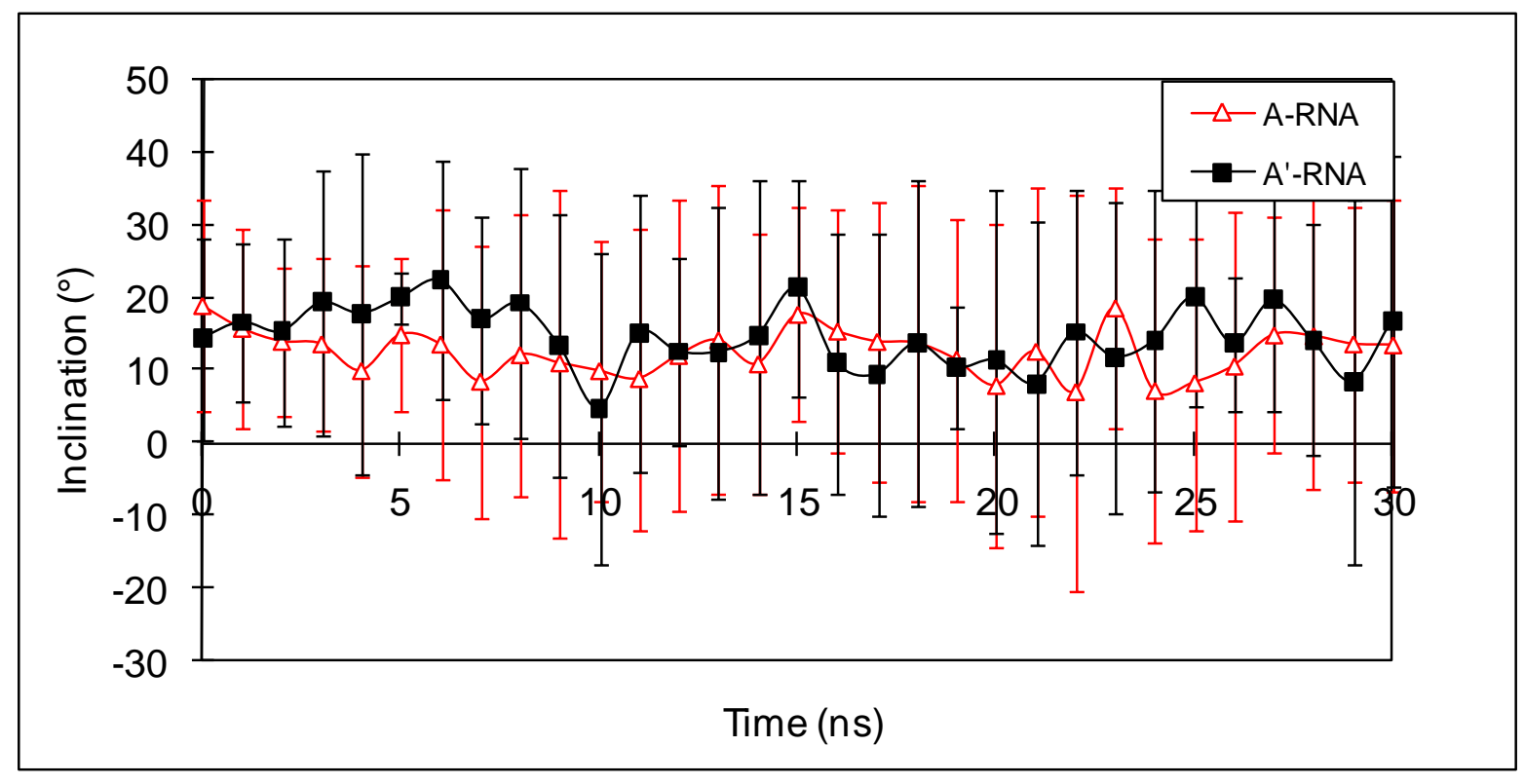

Figure 3b 


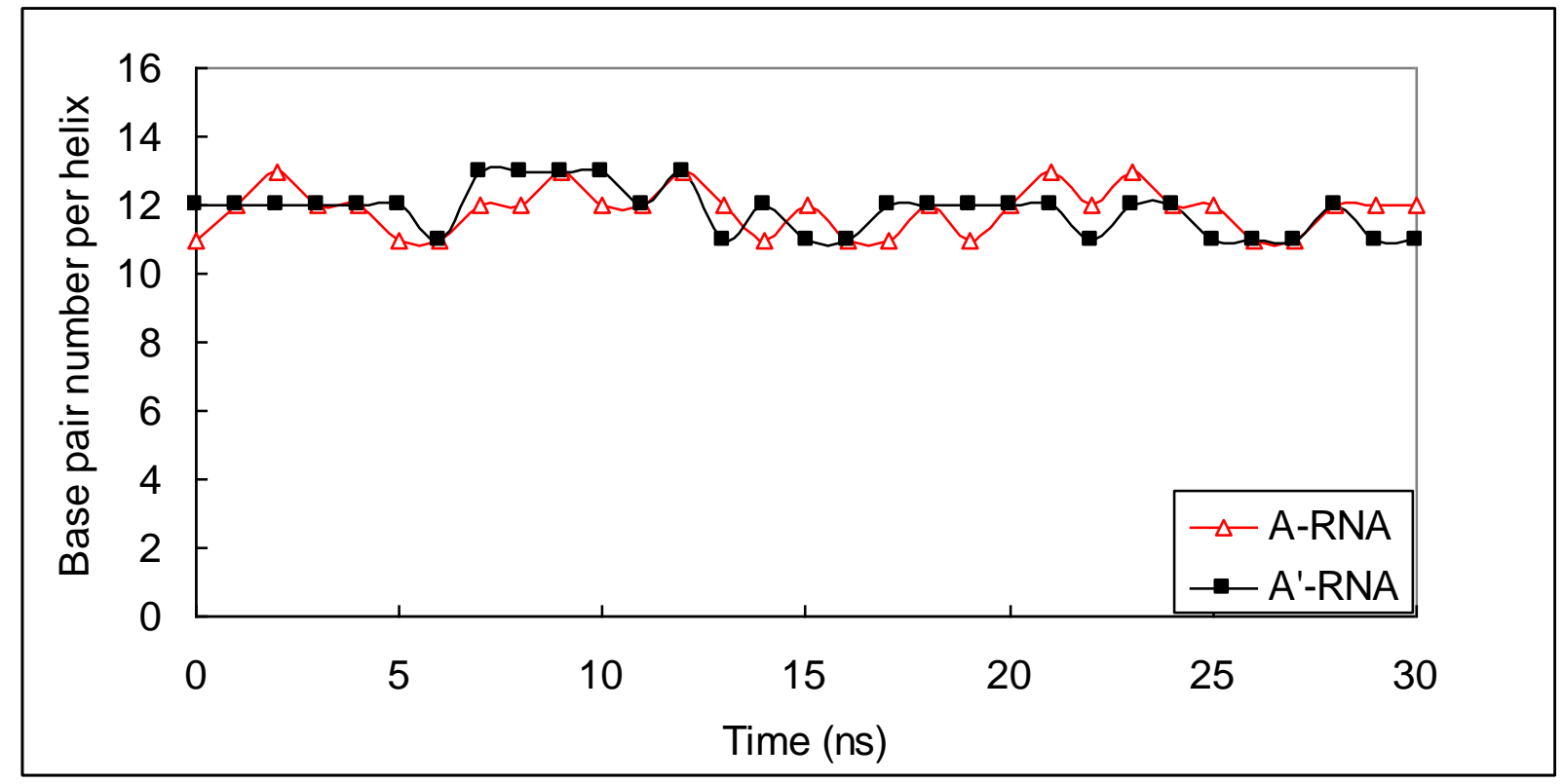

Figure 3c 


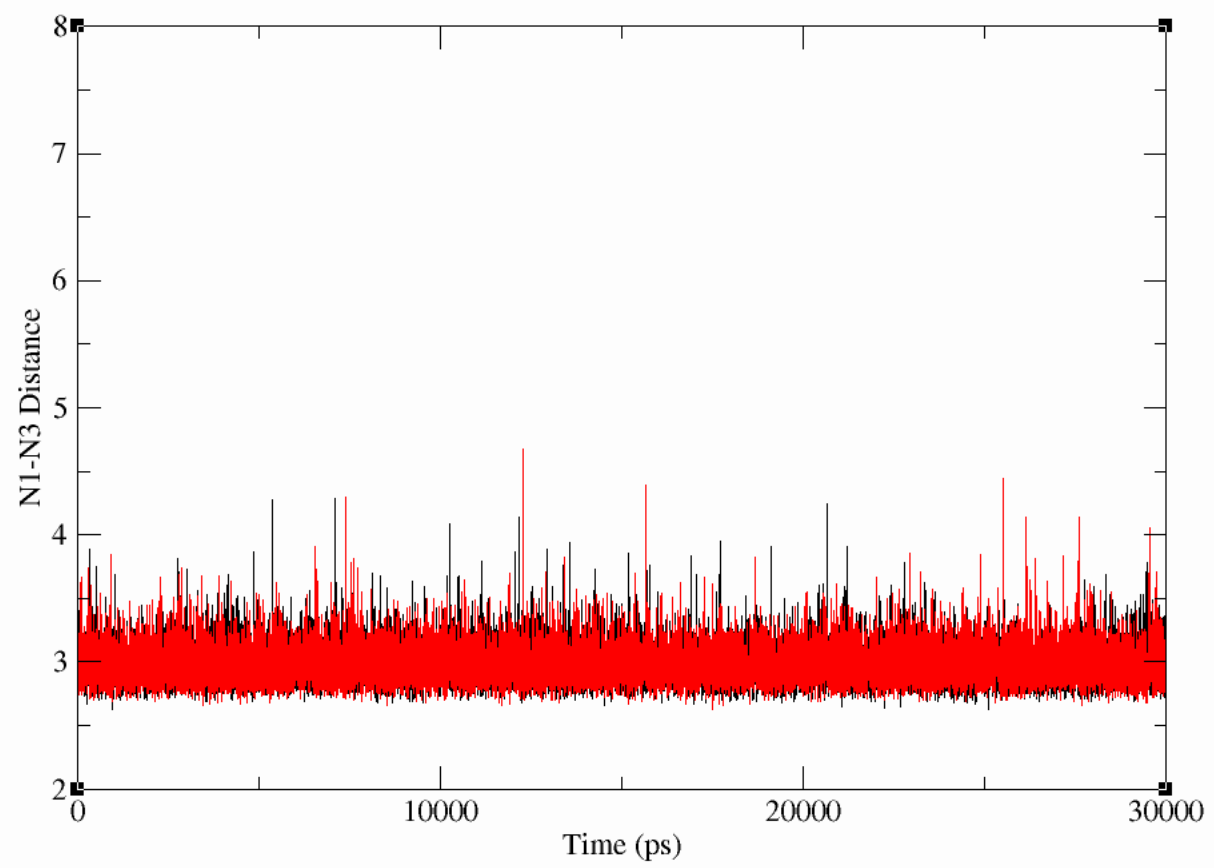

Figure 4a

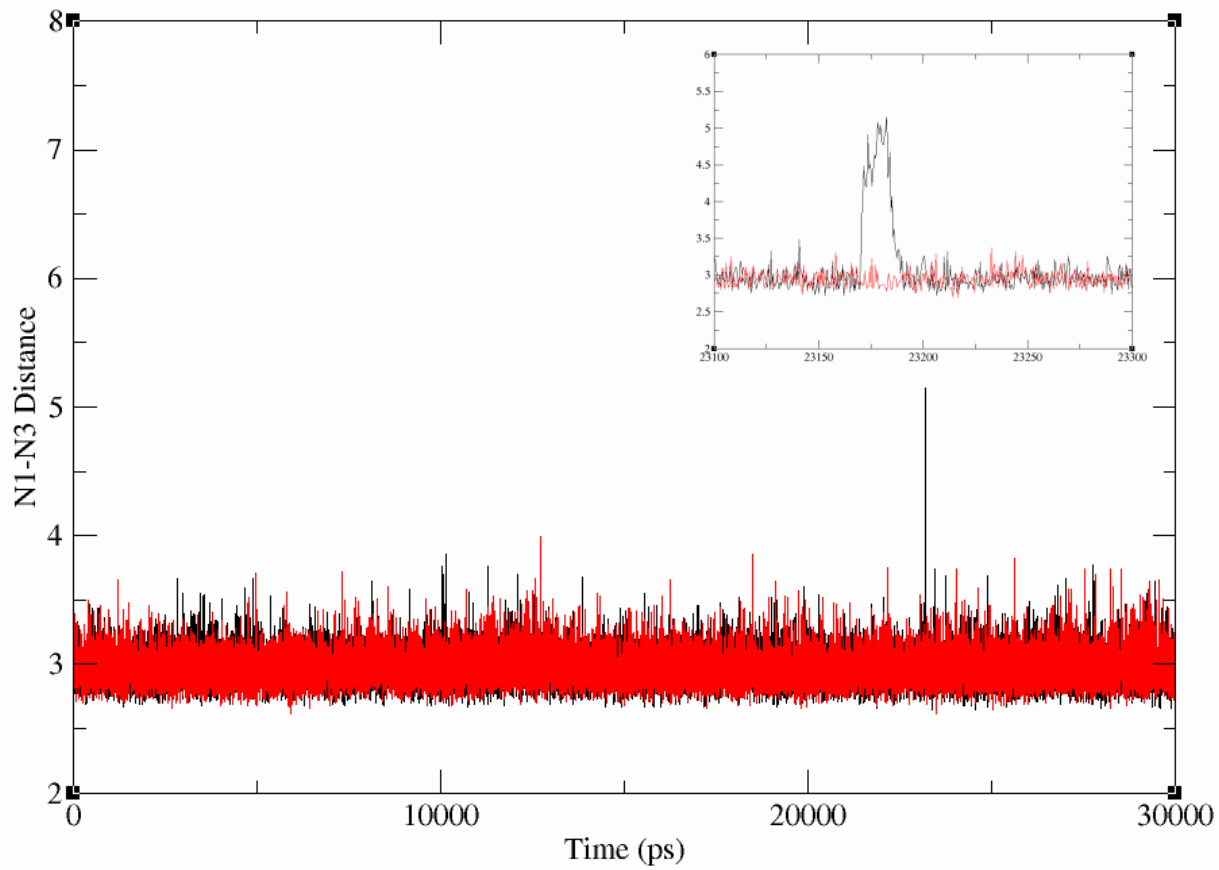

Figure 4b

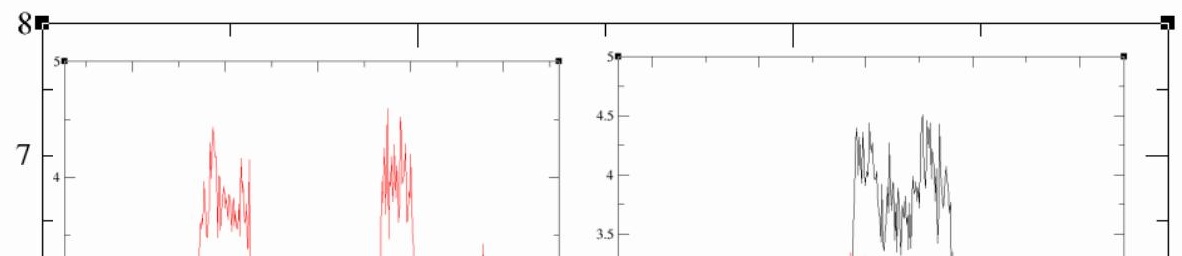


Figure 4c

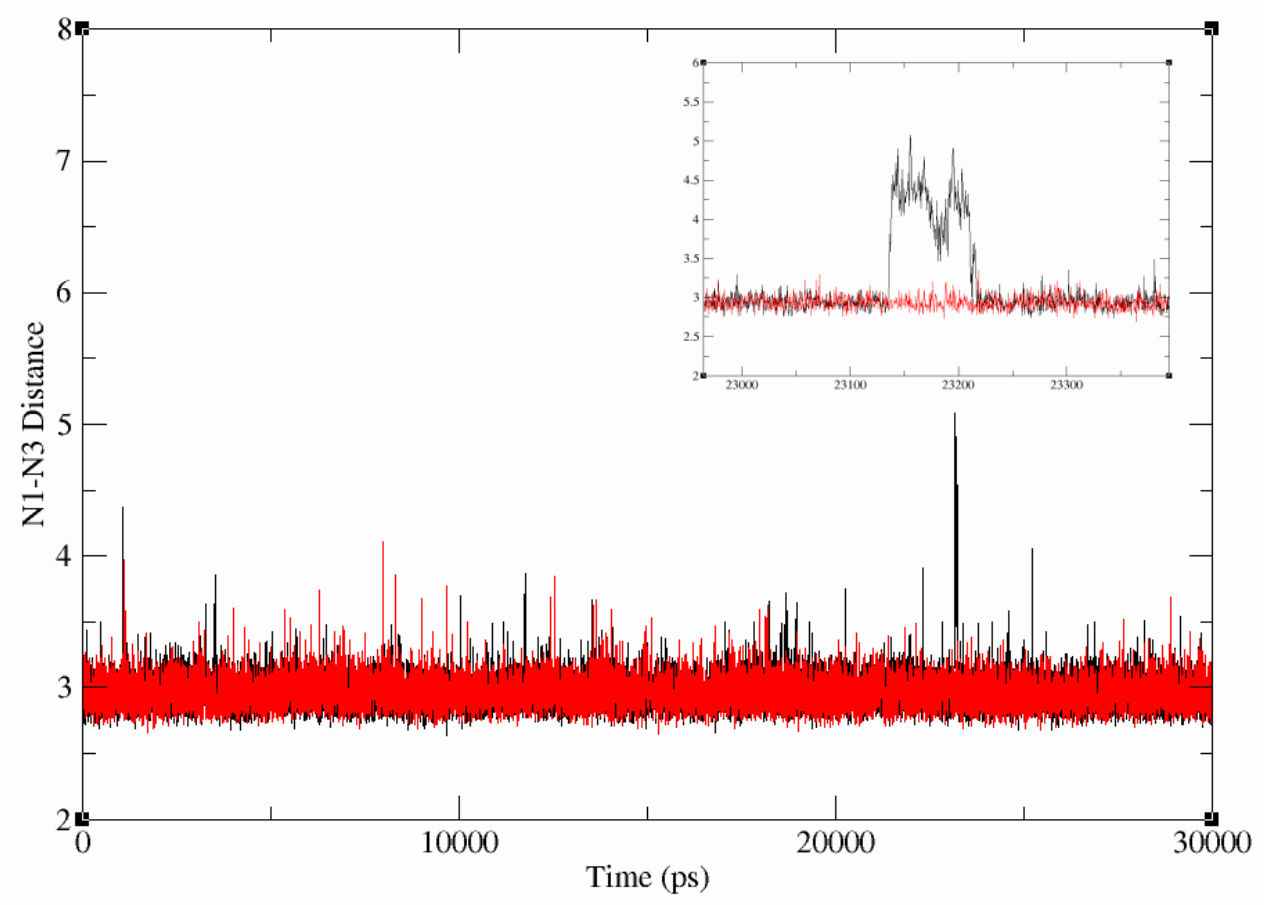

Figure 4d 
Table 1 Comparison of different results by CURVES and 3DNA programs

\begin{tabular}{|c|c|c|c|c|c|c|}
\hline & \multicolumn{2}{|c|}{$413 \mathrm{D}$} & \multicolumn{2}{c|}{$255 \mathrm{D}$} & \multicolumn{2}{c|}{2 ZKO } \\
\hline & CURVES & $3 \mathrm{DNA}$ & CURVES & $3 \mathrm{DNA}$ & CURVES & 3 DNA \\
\hline$x$-disp $(\AA)$ & $-3.25 \pm 0.33$ & $-5.19 \pm 2.46$ & $-3.40 \pm 0.34$ & $-5.39 \pm 2.25$ & $-3.88 \pm 0.32$ & $-4.16 \pm 1.62$ \\
\hline Inclination $\left({ }^{\circ}\right)$ & $-5.00 \pm 2.13$ & $12.60 \pm 8.21$ & $-2.98 \pm 2.51$ & $13.76 \pm 6.20$ & $7.86 \pm 2.68$ & $14.81 \pm 9.89$ \\
\hline Slide $(\AA)$ & $-0.31 \pm 0.43$ & $-1.84 \pm 0.32$ & $-0.40 \pm 0.49$ & $-.199 \pm 0.27$ & $-0.22 \pm 0.38$ & $-1.54 \pm 0.34$ \\
\hline Rise $(\AA)$ & $3.21 \pm 0.29$ & $3.34 \pm 0.17$ & $3.13 \pm 0.24$ & $3.35 \pm 0.16$ & $2.86 \pm 0.18$ & $3.23 \pm 0.13$ \\
\hline Roll $\left(^{\circ}\right)$ & $9.01 \pm 2.12$ & $6.15 \pm 2.69$ & $8.64 \pm 1.40$ & $6.88 \pm 1.87$ & $3.55 \pm 3.66$ & $7.78 \pm 3.94$ \\
\hline Twist $\left({ }^{\circ}\right)$ & $29.80 \pm 5.70$ & $30.60 \pm 8.09$ & $30.31 \pm 6.42$ & $30.84 \pm 9.04$ & $31.59 \pm 3.12$ & $31.02 \pm 5.09$ \\
\hline Stretch $(\AA)$ & $-0.23 \pm 0.51$ & $-0.17 \pm 0.18$ & $-0.29 \pm 0.61$ & $-0.18 \pm 0.21$ & $-0.08 \pm 0.29$ & $-0.24 \pm 0.30$ \\
\hline Stagger $(\AA)$ & $-0.19 \pm 0.16$ & $-0.13 \pm 0.15$ & $-0.11 \pm 0.13$ & $-0.05 \pm 0.12$ & $-0.09 \pm 0.12$ & $0.06 \pm 0.17$ \\
\hline $\begin{array}{c}\text { Minor groove } \\
\text { width }(\AA)\end{array}$ & $9.95 \pm 0.74$ & $11.54 \pm 0.56$ & $9.83 \pm 0.59$ & $11.53 \pm 0.60$ & $10.20 \pm 0.44$ & $11.81 \pm 0.55$ \\
\hline $\begin{array}{c}\text { Major groove } \\
\text { width }(\AA)\end{array}$ & $8.54 \pm 0.25$ & $11.44 \pm 0.52$ & $7.94 \pm 0.28$ & $11.36 \pm 0.51$ & $5.68 \pm 2.19$ & $10.76 \pm 0.93$ \\
\hline
\end{tabular}

Note: The above values show the average structure parameter and standard deviation for each oligomer. The coordinates used her for the calculation of the structure parameters were taken from PDB, the ID number being 413D, 255D, 2ZKO. 
Table 2 Starting structures and average structures in 30 ns of A-RNA and A'-RNA for the backbone angles and helical parameters.

\begin{tabular}{|c|c|c|c|c|}
\hline & \multicolumn{2}{|c|}{ A-RNA } & \multicolumn{2}{c|}{ A'-RNA $^{\text {Average structures }}$} \\
\hline & Starting structures & $\begin{array}{c}\text { Av 30 ns } \\
\text { Starting structures }\end{array}$ & $\begin{array}{c}\text { Average structures } \\
\text { in 30 ns }\end{array}$ \\
\hline$x$-disp $(\AA)$ & $-4.61 \pm 0.28$ & $-4.71 \pm 1.34$ & $-4.72 \pm 0.30$ & $-4.69 \pm 0.67$ \\
\hline Inclination $\left(^{\circ}\right)$ & $16.02 \pm 0.68$ & $15.33 \pm 6.29$ & $9.97 \pm 0.46$ & $18.20 \pm 4.42$ \\
\hline Shift $(\AA)$ & $0.00 \pm 0.02$ & $0.06 \pm 0.18$ & $0.00 \pm 0.02$ & $-0.07 \pm 0.25$ \\
\hline Slide $(\AA)$ & $-1.75 \pm 0.03$ & $-1.73 \pm 0.22$ & $-1.90 \pm 0.04$ & $-1.74 \pm 0.28$ \\
\hline Rise $(\AA)$ & $3.40 \pm 0.01$ & $3.30 \pm 0.13$ & $3.37 \pm 0.01$ & $2.74 \pm 0.19$ \\
\hline Tilt $\left(^{\circ}\right)$ & $0.00 \pm 0.08$ & $0.20 \pm 0.72$ & $0.00 \pm 0.04$ & $-0.08 \pm 0.61$ \\
\hline Roll $\left(^{\circ}\right)$ & $8.91 \pm 0.12$ & $7.81 \pm 3.05$ & $5.14 \pm 0.04$ & $9.56 \pm 2.64$ \\
\hline Twist $\left(^{\circ}\right)$ & $31.55 \pm 1.23$ & $29.13 \pm 2.45$ & $29.62 \pm 1.20$ & $29.25 \pm 1.09$ \\
\hline Shear $(\AA)$ & $0.00 \pm 0.11$ & $0.00 \pm 0.15$ & $0.00 \pm 0.10$ & $-0.12 \pm 0.46$ \\
\hline Stretch $(\AA)$ & $-0.12 \pm 0.06$ & $-0.14 \pm 0.08$ & $-0.12 \pm 0.06$ & $-0.52 \pm 0.21$ \\
\hline Stagger $(\AA)$ & $0.03 \pm 0.00$ & $-0.03 \pm 0.05$ & $0.04 \pm 0.00$ & $-0.01 \pm 0.06$ \\
\hline Buckle $\left({ }^{\circ}\right)$ & $0.00 \pm 0.20$ & $-0.75 \pm 4.33$ & $0.00 \pm 0.22$ & $-0.50 \pm 3.11$ \\
\hline Propol $\left(^{\circ}\right)$ & $13.74 \pm 0.01$ & $-12.73 \pm 2.76$ & $15.11 \pm 0.04$ & $-13.20 \pm 1.57$ \\
\hline Oppening $\left(^{\circ}\right)$ & $-5.52 \pm 0.13$ & $0.90 \pm 1.16$ & $-5.63 \pm 0.09$ & $0.86 \pm 1.58$ \\
\hline
\end{tabular}

